\title{
Rates of cerebrospinal fluid infection and the causative organisms following shunt procedures in Saudi Arabia
}

\author{
A retrospective study based on radiological findings
}

AbdulAziz M. Al-Sharydah, MD, Yaser A. Abu Melha, MD, Sari S. Al-Subibani, MD, Abdulrazaq A. Alojan, MD,

Tareq H. Al-Taei, MD, Iba I. Alfawaz, MD, Lateefah T. AlShammari, MD, Saeed A. Al-Jubran, MD,

Ahmed S. Ammar, MD, PhD.

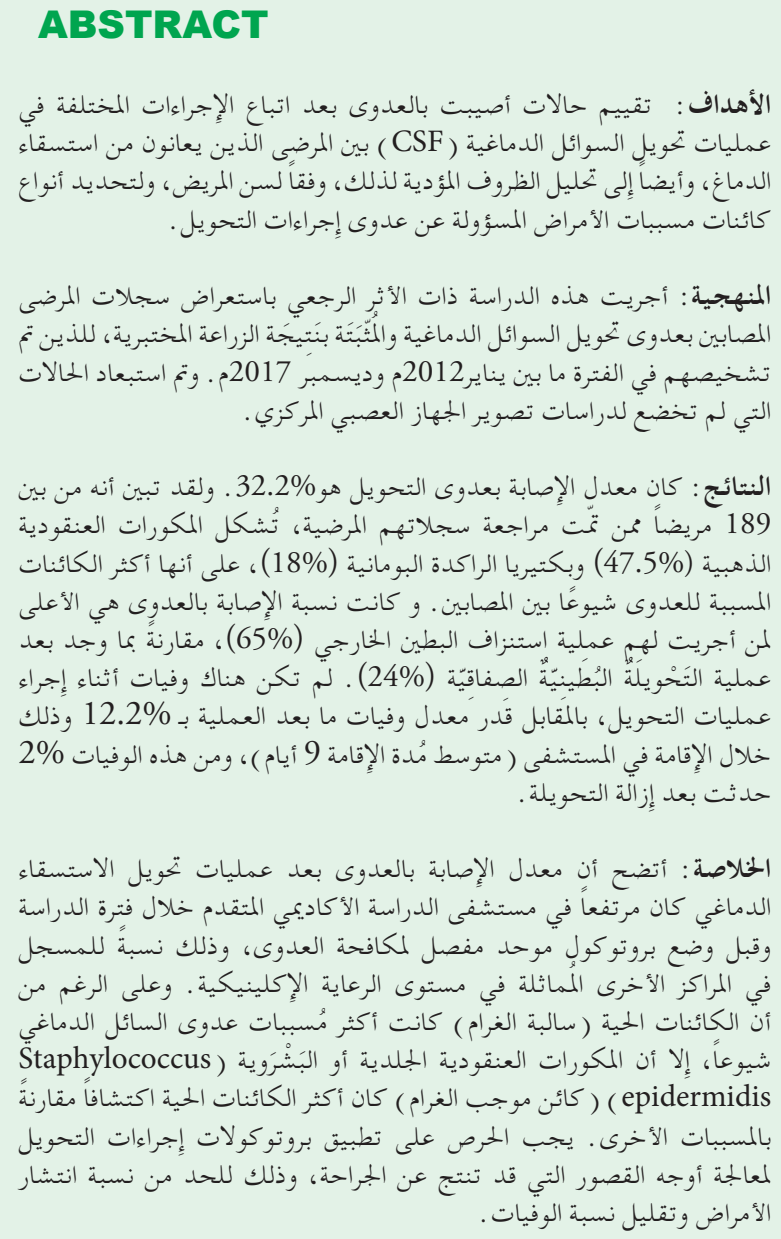

Objectives: To assess the incidence of infection following various cerebrospinal fluid (CSF) shunt procedures among patients with hydrocephalus and related conditions, based on the age of the patient, and to determine the types of pathogens responsible for shunt infections.

Methods: This retrospective single center study reviewed the records of patients with culture-confirmed CSF shunt infection diagnosed between January 2012 and
December 2017. Cases without central nervous system imaging investigations were excluded.

Results: The incidence rate of shunt-related infections was $32.2 \%$. Among the 189 patients whose records were included, Staphylococcus epidermidis (47.5\%) and Acinetobacter baumannii (18\%) were the most common causative organisms. The incidence of postoperative infection was higher among those who underwent external ventricular drain procedures (65\%) than among those who underwent ventriculoperitoneal shunt procedures (24\%). There were no intraoperative deaths. However, the postoperative death rate was $12.2 \%$ during hospital stay (mean duration $=9$ days); $2 \%$ of these deaths occurred after shunt removal.

Conclusion: The incidence of infections after CSF shunt procedures was high in our tertiary academic hospital during the study period, compared to those reported in similar clinical settings and before developing a detailed standardized protocol for infection control. Although gram-negative organisms were the most prevalent cause of CSF infections, S. epidermidis (a gram-positive organism) was the most commonly encountered causative agent. Shunt protocols should be implemented to address perioperative shortcomings and to reduce overall mortality and morbidity.

Keywords: cerebrospinal fluid, shunt-related infections, postoperative infection

\section{Saudi Med J 2020; Vol. 41 (6): 607-613} doi: 10.15537/smj.2020.6.25095

From the Diagnostic \& Interventional Radiology Department (Al-Sharydah, Al-Subibani, Al-Taei, Alfawaz, Al-Jubran); from the Neurosurgery Department (Ammar, Alojan, Lateefa), Imam Abdulrahman Bin Faisal University, King Fahd Hospital of the University, AlKhobar; and from the Microbiology Department (Melha), Security Forces Hospital, Riyadh, Kingdom of Saudi Arabia.

Received 21st January 2020. Accepted 27th January 2020.

Address correspondence and reprint request to: Dr. Sari S. Al-Suhibani, Consultant of Neuroradiology. Diagnostic and Interventional Radiology Department, King Fahd Hospital of the University, Imam Abdulrahman Bin Faisal University, AlKhobar, Kingdom of Saudi Arabia. E-mail: ssubibani@iau.edu.sa

ORCID ID: https://orcid.org/0000-0002-4480-994X 
$\mathrm{H}_{\mathrm{i}}^{\mathrm{y}}$ ydrocephalus is a commonly observed condition in neurosurgical practice; it requires cerebrospinal fluid (CSF) diversion. In Saudi Arabia, the incidence of hydrocephalus is higher than that in most other countries, because of the high rate of marriages and the prohibition of abortion. ${ }^{1}$ The national prevalence of congenital hydrocephalus is estimated to be 0.8 to 1.6 per 1,000 live births. ${ }^{1-3}$ Although the primary complications of shunt procedures include obstruction and drainage problems, postsurgical infections are the most common, immediate, life-threatening complications. ${ }^{1,4,5}$

Shunt infections are usually caused by nosocomial organisms as well as community-acquired pathogens; they cause clinically significant morbidity and mortality rates worldwide. ${ }^{6}$ The rate of infection following CSF diversion procedures ranges from $4 \%$ to $30 \% .^{7}$ In the United States, infection rates range from $4.1 \%$ to $17.4 \%$ of patients and from $2.5 \%$ to $12.3 \%$ based on the type of CSF diversion procedure conducted. ${ }^{8}$ In countries like the Arabian Peninsula, figures on the incidence of shunt infections are scarce and vary from $3 \%$ to $52 \%$.,4

Typically, shunt infections occur 15 days to 12 months following surgery. Most shunt infections are caused by gram-positive organisms, with coagulase-negative staphylococci causing 17-78\% and Staphylococcus aureus causing $44-30 \%$ of shunt infections. ${ }^{9}$ The symptoms of postoperative shunt infections include the following: a mild or, rarely, severe fever; nausea; vomiting; malaise; and lethargy and a loss of appetite, especially when the infection develops into bacteremia. Shunt infections are often associated with wound dehiscence, which causes patients to revisit healthcare facilities. ${ }^{10}$

The type of surgery in which the surgical incision passes pathogens from the skin to internal body organs (such as the brain) is one of the essential causes of shunt infections. Furthermore, the infection might spread locally from one site to another via either hematogenous, airborne, or direct contact with the source. ${ }^{6}$ The use of ventriculoperitoneal shunt (VPS), external ventricular drain (EVD), or endoscopic third ventriculostomy (ETV) in patients with hydrocephalus divert CSF from the ventricles to the peritoneum and collecting system or to the basal tank. This allows for an opportunity for transmission of infections to the central nervous system

Disclosure. Authors have no conflict of interests, and the work was not supported or funded by any drug company.
(CNS), resulting in conditions such as meningitis and cerebral abscesses. ${ }^{11}$

This study aim to determine the incidence rate of shunt infections and the primary causative organisms among patients who underwent shunt surgery in a tertiary academic hospital in Saudi Arabia. Additionally, we aim to review the patients' clinical, radiological, and microbiological features.

Methods. A cross-sectional retrospective review was conducted among patients treated for CSF shunt infections at, a 600-bed tertiary academic public hospital, King Fahd Hospital of the University, Dammam, Saudi Arabia, between January 2012 and December 2017. Ethical approval was obtained from the Institutional Review Board of Imam Abdulrahman Bin Faisal University. As a result of the retrospective nature of the study, the need for informed consent was waived. This study was conducted according to the principles of the Helsinki Declaration.

Our literature search was extensive, comprehensive, and was only influenced by study quality and suitability. Data were obtained from patients' electronic medical records. The International Classification of Diseases (ICD-9) keyword "CNS infections" was used to identify the electronic medical files of patients treated for CNS infections. Next, we reviewed the hospital database to identify cases of shunt infection that occurred during the 6-year study period. Data on patient demographics, underlying conditions, causative microorganisms, type of shunt device, and the time between surgery and diagnosis were extracted. Infections were categorized as early ( $<1$ month after shunt surgery), delayed (1 to 12 months after surgery), or late ( $\geq 12$ months after surgery).

Patients were included in the study if they had a positive microbiological culture within 120 hours after surgery, a postoperative fever (temperature $>38.2^{\circ} \mathrm{C}$ ), and available CNS imaging data. Cases of shunt infection in which the shunt was removed before determination of the causative organism and cases in which the patients were diagnosed in different health facilities were excluded.

Statistical analysis. Statistical analyses were performed using Statistical Package for Social Sciences, version 21.0 (IBM Corp, Armonk, NY, USA). Descriptive results were reported as frequencies and percentages. Comparisons between groups were performed using analysis of variance, and the KruskalWallis test was used to test the significance of differences between groups. $P$-values $<0.05$ were considered statistically significant. 
Results. Of the 266 patients who underwent a CNS shunt procedure, 189 (71.1\%) met the inclusion criteria. Of the patients with shunt infections, 32.3\% had a positive CSF culture (Table 1).

The incidence of infection was the highest among patients with an EVD (29/52, 56\%), followed by patients with an VPS $(31 / 131,24 \%)$, and an ETV (1/6, $15 \%)$. The incidence of infection differed significantly based on the type of procedure conducted $(p=0.02)$. The incidence of infection was the highest among patients with post-traumatic hydrocephalus (4/8, $50 \%)$. The rate of infection was also high among those with congenital hydrocephalus (29\%), spina bifida with hydrocephalus (33\%), and hydrocephalus secondary to a vascular abnormality (45\%); however, the incidence of infection did not differ significantly based on the type of underlying condition (Table 2).

The majority of shunt infections $(52.8 \%)$ were caused by gram-negative microorganisms. Gram-positive

Table 1 - Incidence of shunt infections based on age.

\begin{tabular}{lccc}
\hline Age group & $\begin{array}{c}\text { Positive } \\
\text { culture }\end{array}$ & $\begin{array}{c}\text { Negative } \\
\text { culture }\end{array}$ & $\begin{array}{c}\text { Total } \\
\text { incidence } \\
\mathbf{n}(\%)\end{array}$ \\
\hline Neonate (<1 month) & 1 & 12 & $13(8)$ \\
Infant (1-11 months) & 8 & 21 & $29(28)$ \\
Pre-school (1-5 years) & 16 & 20 & $36(44)$ \\
Children (6-12 years) & 5 & 23 & $28(18)$ \\
Adolescent (13-18 years) & 5 & 19 & $24(21)$ \\
Adults (19-64 years) & 21 & 31 & $52(40)$ \\
Older adults (>65 years) & 5 & 2 & $7(71)$ \\
\hline
\end{tabular}

Table 2 - Incidence of shunt infections based on the diagnosis at admission.

\begin{tabular}{lc}
\hline Diagnosis & $\begin{array}{c}\text { Incidence of infections/ } \\
\text { procedures } \\
\text { n/n }(\%)\end{array}$ \\
\hline $\begin{array}{l}\text { Congenital hydrocephalus } \\
\text { Spina bifida with hydrocephalus }\end{array}$ & $11 / 72(29)$ \\
Hydrocephalus secondary to a & $11 / 24(43)$ \\
vascular abnormality & \\
Obstructive hydrocephalus & $6 / 20(30)$ \\
secondary to a space-occupying & \\
lesion & $2 / 9(22)$ \\
Dander-walker syndrome with & $4 / 8(50)$ \\
hydrocephalus & $2 / 6(33)$ \\
Post-traumatic hydrocephalus & $1 / 6(16)$ \\
Arachnoid cyst with hydrocephalus & $2 / 5(40)$ \\
Post-infectious hydrocephalus & $1 / 3(33)$ \\
Intraventricular hemorrhage of & $0 / 3(0)$ \\
prematurity & \\
Colloid cyst with hydrocephalus & Normal pressure hydrocephalus
\end{tabular}

bacterial caused $44 \%$ and fungal pathogens caused $3.3 \%$ of the infections. Among the 61 cases of bacterial infections, Staphylococcus epidermidis (S. epidermidis) was the most common cause of infection (29/61, 48\%), followed by Acinetobacter baumannii (11/61, 18\%) (Figures 1 \& 2 ), (Table 3). Less frequently cultured microorganisms included group A beta-hemolytic Streptococci, group B Streptococci, group B Aspergillus, Proteus mirabilis, Pseudomonas species, and S. hominis.

There were no intraoperative deaths. However, the postoperative death rate was $12.2 \%$ during hospital stay; $2 \%$ of postoperative deaths occurred after the shunt was removed. The mean duration of hospital stay was 9 days.

Discussion. The burden of infantile hydrocephalus in Saudi Arabia, a developing country, is significantly higher than those in other developed countries, including Sweden, England, Wales, and Japan. The incidence of congenital hydrocephalus in Saudi Arabia is estimated to be 0.81 to 1.6 per 1000 live births. ${ }^{1-3}$

In this study, the incidence of shunt infections exceeded $32 \%$, which is significantly higher than that reported in similar clinical settings. ${ }^{9,10}$ Additionally, the causative organisms differed from those found in previous studies conducted in Saudi Arabia, with some new pathogens, such as Serratia marcescens, being identified. $^{3}$ Thus, management of the factors that contribute to the high rate of shunt infections; such as hygiene, wound care by the patient or the nursing staff, duration of hospital admission, experience of the surgeon, surgical technique used, duration of the procedure, and handling of the indwelling device during surgery; and the more frequent use of antibiotics than that in other countries are required. However, this may lead to the emergence of antibiotic resistance, thus limiting antibiotic choices and efficacy in the future.

Use of VPS is known to be associated with a higher rate of infection than the use of other methods of CSF diversion. ${ }^{11}$ However, in our study, the rate of infection was higher following EVD procedures, despite VPS procedures being the most commonly performed procedure. It has been reported that the incidence of postsurgical VPS infection ranges from 3\% to $9 \%$ in Western countries and from $9 \%$ to $32 \%$ in African countries; the incidence is lower in developed countries. ${ }^{12}$ In countries in sub-Saharan Africa, especially in regions with endemic bacterial infections, it is advisable to use empirical broad-spectrum antibiotics to prevent postoperative infection. ${ }^{13}$ Until recently, the use and effects of prophylactic antibiotics have been a source of debate. ${ }^{12,13}$ For the best results, it is essential to 
Table 3 - Key distinguishing radiological and microbiological features of common causative microbes associated with shunt infections.

\begin{tabular}{|c|c|c|}
\hline Pathogen & Key radiological features & Key microbiological features \\
\hline Staphylococcus epidermidis & Meningitis, hydrocephalus, IVH & $\begin{array}{l}\text { Positive: gram staining/capsule/Catalase/ } \mathrm{H}_{2} \mathrm{~S} / \text { gas/Urease } \\
\text { Negative: Citrate/Coagulase/GH/Oxidase/hemolysis }\end{array}$ \\
\hline Acinetobacter baumannii & $\begin{array}{l}\text { Large hematoma, IVH, } \\
\text { hydrocephalus, periventricular } \\
\text { interstitial edema, and occipital horn } \\
\text { enhancement (intracranial extension) }\end{array}$ & $\begin{array}{l}\text { Positive: capsule/Catalase/Citrate } \\
\text { Negative: gram staining (coccobacilli)/Coagulase/gas/GH/H } 2 \mathrm{~S} / \text { hemolysis/ } \\
\text { motility/Flagella/MR/Nitrate/OF/Urease }\end{array}$ \\
\hline Enterococcus faecalis & $\begin{array}{l}\text { Abscess with gas and subdural } \\
\text { empyema }\end{array}$ & $\begin{array}{l}\text { Positive: gram staining/OF/PYR } \\
\text { Negative: capsule/Flagella/Catalase/Citrate/ } \mathrm{H}_{2} \mathrm{~S} / \mathrm{Gas} / \text { Nitrate/Urease }\end{array}$ \\
\hline Enterobacter cloacae & $\begin{array}{l}\text { Abscess, meningitis, ventriculitis, } \\
\text { pseudomeningoceles, } \\
\text { pneumocephalus, IVH, interstitial } \\
\text { edema, and encephalomalacia }\end{array}$ & $\begin{array}{l}\text { Positive: catalase/Citrate/Flagella/Gas/OF } \\
\text { Negative: gram staining/Capsule/ } \mathrm{H}_{2} \mathrm{~S} / \mathrm{GH} / \text { Urease }\end{array}$ \\
\hline Pseudomonas aeruginosa & $\begin{array}{l}\text { Abscess, cerebritis, ventriculitis, } \\
\text { mycotic aneurysm, otitis externa, and } \\
\text { osteomyelitis }\end{array}$ & $\begin{array}{l}\text { Positive: Citrate/motility/Oxidative/gas/GH } \\
\text { Negative: gram staining rods/capsule/Catalase/Coagulase/single Fagella/MR/ } \\
\text { Urease/ } \mathrm{H}_{2} \mathrm{~S}\end{array}$ \\
\hline Klebsiella pneumoniae & $\begin{array}{l}\text { Meningitis with or without nodal } \\
\text { enhancement and subdural abscesses }\end{array}$ & $\begin{array}{l}\text { Positive: capsule/catalase/Citrate/Flagella/GH/ } \mathrm{H}_{2} \mathrm{~S} / \text { Urease } \\
\text { Negative: gram staining/gas/motility }\end{array}$ \\
\hline
\end{tabular}

GH: gelatin hydrolysis, H2S: hydrogen sulfide, IVH: intraventricular hemorrhage, KCN: potassium cyanide, MR: methyl red, OF: oxidative/fermentative, PYR: pyrrolidonyl aminopeptidase

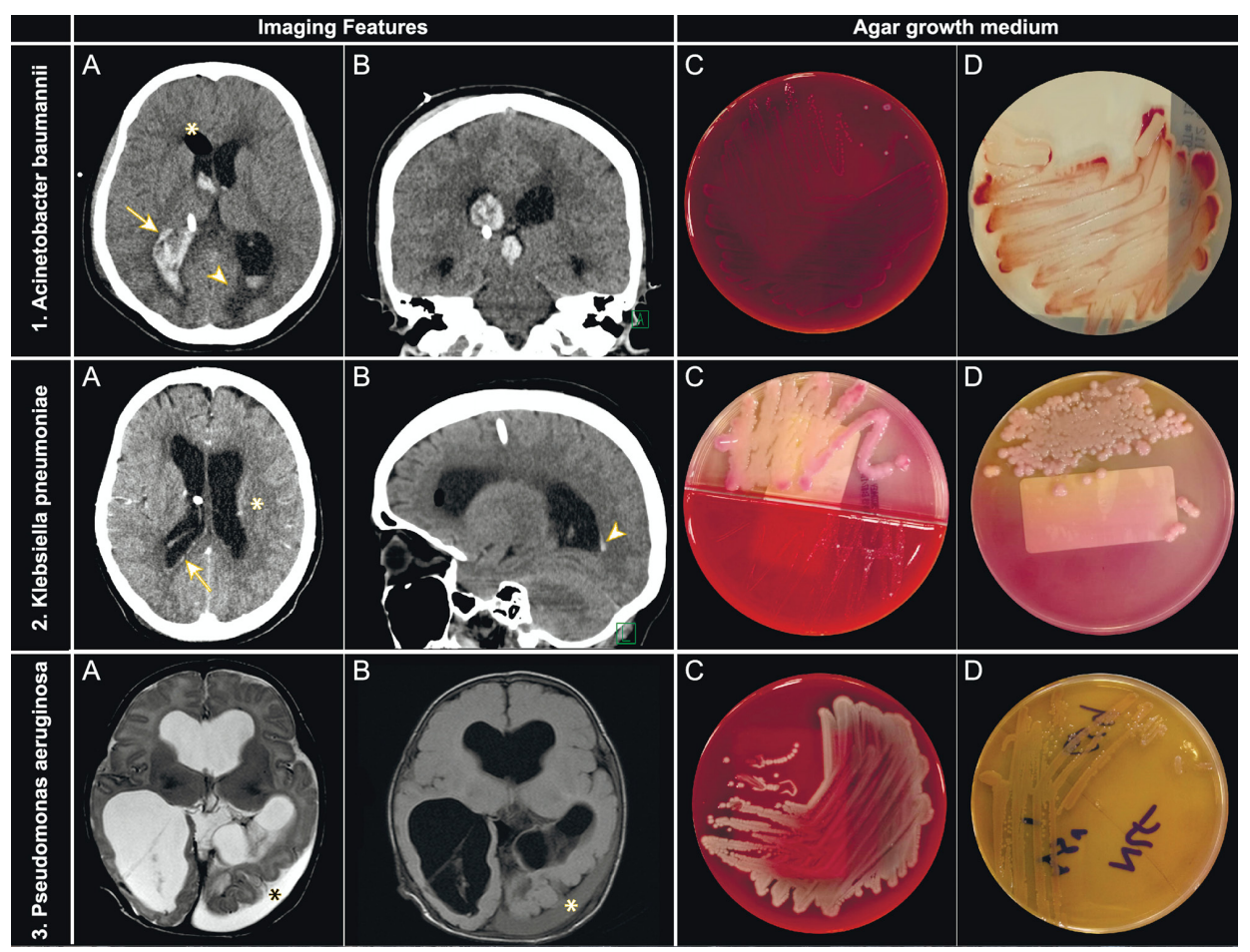

Figure 1 - Examples of distinct radio-microbiological features of shunt-related infections based on the microbial pathogen images (A) and (B) show the brain computed tomography (CT) scans and images (C), and (D) show the appearance of the culture on agar growth medium. 1) Acinetobacter baumannii (A) plain axial and (B) coronal brain CT for extraventricular drain (EVD) follow-up depict persistent ventriculomegaly and postoperative pneumocephalus $\left({ }^{*}\right)$ with the interim development of large intraventricular hemorrhage (IVH) (arrow) and periventricular interstitial edema (arrowhead). (C) Microbiological culture yielded strains that grew well on blood agar- and (D) on CHROMagar (CHROMagar, Paris, France) as red colonies. 2) Klebsiella pneumoniae (A) enhanced axial and (B) plain sagittal brain CT for EVD follow-up depict cerebritis $\left({ }^{*}\right)$, ventriculitis (arrow), and trivial IVH (arrowhead). (C) Microbiological culture yielded gram-negative bacilli that grew well on blood agar and (D) as mucoid pink colonies on MacConkey agar. 3) Pseudomonas aeruginosa Axial T2WI (A) and fluid-attenuated inversion-recovery (B) magnetic resonance imaging of the brain for ventriculoperitoneal shunt follow-up depicting a left temporooccipital subdural abscess $(*)$. (C) Microbiological culture yielded gram-negative non-fastidious rods that grew well on blood agar and (D) were oxidase positive. Colorless colonies are observed on MacConkey agar. The organisms produced water soluble pigments known as pyocyanin /blue-green pigment. 


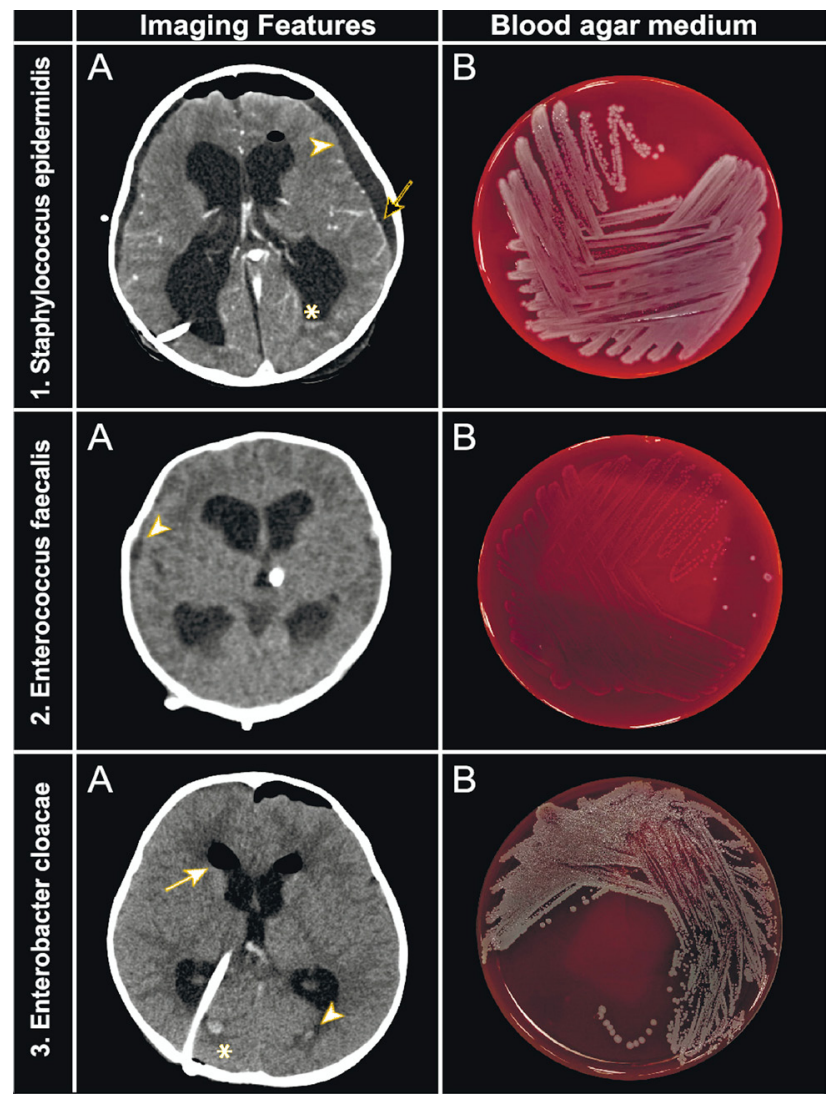

Figure 2 - Examples of distinct radio-microbiological features of infective pathogens and their growth on citrated sheep blood agar. 1) Staphylococcus epidermidis (A) enhanced axial brain computed tomography (CT) for extraventricular drain (EVD) follow-up depicts persistent ventriculomegaly meningoencephalitis (arrow), a subdural abscess (arrowhead), a trivial intraventricular hemorrhage (IVH) $\left(^{*}\right)$, and developing pneumocephalus. (B) Microbiological culture yielded coagulase-negative, gram-positive staphylococci that grew well on sheep blood agar, appeared as white colonies on blood agar, and showed no hemolysis. 2) Enterococcus faecalis (A) plain axial brain CT for EVD follow-up depicts subdural abscess (arrowhead) and IVH $\left({ }^{*}\right)$. (B) Microbiological culture yielded gram-positive, non-hemolytic streptococci that grew on blood agar and were able to hydrolyze esculin in the presence of bile. Enterococcus species are able to grow in the presence of $6.5 \% \mathrm{NaCl}$. 3) Enterobacter cloacae (A) plain axial brain CT for a ventriculoperitoneal shunt follow-up depicts IVH (arrowhead), interstitial edema $(*)$, and pneumocephalus (arrow). (B) Microbiological culture yielded gram-negative, lactose-fermenting bacilli that formed white colonies with a glossy, translucent appearance. On MacConkey agar, these bacilli performed reddish-pink mucoid colonies.

target the most commonly implicated pathogens when administering prophylactic antibiotics before patients undergo surgery.

Sarguna and Lakshmi ${ }^{14}$ retrospectively analyzed the records of 226 patients who had undergone shunt procedures and found only 9 shunt infections (overall infection rate: 4\%); the other CSF and blood cultures were sterile. Their study results indicated that CSF cultures may be negative even when the shunt device harbors infection. ${ }^{15}$

Staphylococcus species are mainly present on the skin and superficial areas; hence, it is difficult to prevent infections cause by these bacteria. ${ }^{10}$ This explains why surgical incisions are prone to staphylococcal infections. Staphylococcus epidermidis, which is a part of the normal skin flora, was the most commonly encountered organism in our study; this is in keeping with reported international figures. Staphylococcus epidermidis is a permanent and pervasive colonizer of human skin, and there is a high chance of device contamination during insertion through the scalp skin (Figure 2). ${ }^{16,17}$

Although most (90\%) shunt infections occur within 20 days of surgery, some infections may not occur until many years after surgery. ${ }^{10}$ Viereck et al ${ }^{15}$ reported that symptoms of cryptococcal infection started 10 days to 15 months after shunt placement. They also reported a case involving a VPS procedure in which a cryptococcal infection was only detected 20 years after VPS placement. This type of infection is an infrequent complication of VPS, and only 9 cases of shunt-related cryptococcal infections have been reported to date. ${ }^{18}$ Having said that, our results did not reveal any cases of cryptococcal infection.

Based on imaging findings, the majority of CNS infections can be classified based on their MR sequences T1 and T2 weighted imaging and contrast enhancement characteristics. Ring enhancing lesions, basal ganglia space-occupying lesions, and gray and white matter hyperintensities are common patterns. Imaging is of substantial importance to detect the signs of infection caused by pyogenic bacteria in the subarachnoid space, brain parenchyma, ventricles (ventriculitis or pyocephalus), and the potential extra-axial spaces (epidural or subdural empyema). Similar to parenchymal abscesses, pus in the ventricles (occipital horns) or epidural or subdural space shows restricted diffusion, a useful finding for distinguishing simple effusion from empyema in the context of acute bacterial meningitis (Figures 1 \& 2 ). Magnetic resonance spectroscopy is useful to evaluate necrotic masses; for example, infections with a central necrotic cavity lacking normal brain metabolites (choline, creatine, and n-acetyl aspartate) can be evaluated using this technique. Restricted diffusion or periventricular enhancement depends on whether the patient can mount an inflammatory response against pathogens (Table 3). ${ }^{19-26}$ 
Cerebrospinal fluid shunt infections caused by Candida albicans (C. albicans) are rare because this fungal agent only thrives well among individuals in immunosuppressed states. ${ }^{18}$ Cancer patients, those receiving chemotherapy, or individuals with HIV infection are at higher risk of these CSF shunt infections. ${ }^{12}$ In this study, C. albicans was identified in CSF cultures from immunocompetent patients $(2.5 \%)$, even though it is difficult to isolate $C$. albicans from immunocompetent patients in the laboratory. ${ }^{27}$

The incidence of CSF shunt infections varies with age; it has been reported to be the highest among infants and to decline with increasing age. ${ }^{12,14}$ However, this was not the case in our study. The rate of infection was the highest in elderly patients, adults, and pre-school children. This observation concurs with the proposed factors attributing to postoperative infections, including patient age, immune status, environmental exposure, and nutritional status. ${ }^{28}$ Environmental exposure is the presence of microorganisms that can cause infection. Age is closely related to the immune status. This explains why the least virulent organisms affect children and elderly patients more frequently than the rest of the population.

Cerebrospinal fluid infection is a major concern in the neurosurgical field due to the following reasons: i) adverse cognitive outcomes; ii) high rates of morbidity and mortality; ii) need for prolonged courses of antibiotics following hospitalization; iv) negative psychosocial impact on patients and families; and v) need for shunt revisions. The fact that there is a foreign shunt placed in the body significantly contributes to the development of CSF infections among patients undergoing procedures involving incisions in the ventricular system. ${ }^{28}$ Insertion of shunt devices requires proper diagnosis and handling; therefore, only sterilized hardware (sterilized by steam, ethylene oxide, and other low-temperature sterilizers) should be used.

Precautions are needed to reduce the rate of CSF infections. Bacteremia must be ruled out based on results of complete white blood cell count, inflammatory markers, and other laboratory procedures. Additionally, if patients and physicians adhered to infection control, bacteremia is less likely to occur. Additionally, attempts should be made to minimize the number of operative room personnel, shorten the operative time, and ensure proper instrument sterilization. ${ }^{28}$ Recently, a detailed standardized protocol was published for use during CSF shunt surgeries to prevent postoperative infection regardless of the patient's age. ${ }^{29}$ The protocol includes guidance on the control of the surgical environment to minimize contamination from air, double gloving, local injection of antibiotics, and detailed postoperative management. The protocol also provides guidance on immersion of devices, optimal timing of skull $x$-ray, and detailed postoperative management.

Furthermore, at our facility, we advise strict follow-up at the outpatient clinic within 2 weeks of discharge, to assess the surgical wound margins, CSF culture results from the shunt aspirate, blood test, and culture results. Patient attendance at the clinic is significantly influenced by their health-seeking behaviors, which may influence the development of postoperative complications. Thus, we employed a number of strategies to ensure that patients attended their follow-up appointments, such as scheduling in-person follow-up care appointments at the patient's convenience. Additionally, we attempted to adhere to the core principle of providing the patient or the patient's parents' with comprehensive health education, including information on the health condition and possible complications.

Study limitations. This was a retrospective study conducted at a single center, with a small sample size. As this retrospective study relied on accurate data collection and documentation, interpretation may have been limited by the validity and generalizability of our findings either by information or election bias. Also, this limited our ability to assess the age-specific incidence of infection. Additionally, we did not investigate some confounding factors that may influence our findings, such as the incidence of multiple infections in the same patient.

In conclusion, the incidence of infection after CSF shunt procedures was high in our hospital during the study period, and was significantly higher than the rate observed in other similar clinical settings before developing a detailed standardized protocol for infection control. Although gram-negative organisms were the most common cause of CSF infections, S. epidermidis (a gram-positive organism) was the single most frequently encountered organism. The postoperative infection rate was higher following EVD procedures than following VPS procedures, despite the fact that the VPS procedure was more commonly used. Shunt protocols should be implemented to address perioperative shortcomings and to reduce overall hospital stay, mortality and morbidity.

Emphasizing and encouraging the surgeons on giving the patients clear instructions and educating them on infection control measures might help in decreasing the infection rate. Further study is needed to be conducted in order to formulate an optimal management strategy for these patients.

Acknowledgment. The authors gratefully acknowledge Editage (www.editage.com) for English language editing. 


\section{References}

1. Al Anazi AR, Nasser MJ. Hydrocephalus in eastern province of Saudi Arabia. Qatar Med J 2003; 2003: 19.

2. Murshid WB, Jarallah JS, Dad MI. Epidemiology of infantile hydrocephalus in Saudi Arabia: Birth prevalence and associated factors. Pediatr Neuro Surg 2000; 32: 119-123.

3. El Awad ME. Infantile hydrocephalus in the south-western region of Saudi Arabia. Ann Trop Pediatr 1992; 12: 335-338.

4. Obeid F. Review of hydrocephalus treated by ventriculoperitoneal shunt and role of local Vancomycin injection in treatment of shunt infection. Pan Arab J Neurosurg 1997; 1: 54-57.

5. Dupepe EB, Hopson B, Johnston JM, Rozzelle CJ, Oakes WJ, Blount JP, et al. Rate of shunt revision as a function of age in patients with shunted hydrocephalus due to myelomeningocele. Neurosurg Focus 2016; 41: E6.

6. Bin Nafisah S, Ahmad M. Ommaya reservoir infection rate: A 6-year retrospective cohort study of Ommaya reservoir in pediatrics. Childs Nerv Syst 2015; 31: 29-36.

7. Stadler JA, Aliabadi H, Grant GA. Complications associated with cerebrospinal fluid diversion. In: Nanda A, editor. Complications in Neurosurgery. Amsterdam (NH): Elsevier; 2019. p. 172-177.

8. Simon TD, Hall M, Riva-Cambrin J, Albert JE, Jeffries HE, Lafleur B, et al. Infection rates following initial cerebrospinal fluid shunt placement across pediatric hospitals in the United States. J Neurosurg Pediatr 2009; 4: 156-165.

9. Bremer AA, Darouiche RO. Ventriculoperitoneal shunt infection due to Serratia marcescens. J Infect 2005; 50: 138-141.

10. Duhaime AC. Evaluation and management of shunt infections in children with hydrocephalus. Clin Pediatr (Phila) 2006; 45: 705-713.

11. Chu JK, Sarda S, Falkenstrom K, Boydston W, Chern JJ. Ventricular access device infection rate: a retrospective study and review of the literature. Childs Nerv Syst 2014; 30: 1663-1670.

12. Ochieng' N, Okechi H, Ferson S, Albright AL. Bacteria causing ventriculoperitoneal shunt infections in a Kenyan population. J Neurosurg Pediatr 2015; 15: 150-155.

13. Bokhary MA, Kamal H. Ventriculo-peritoneal shunt infections in infants and children. Libyan J Med 2008; 3: 20-22.

14. Sarguna P, Lakshmi V. Ventriculoperitoneal shunt infections. Indian J Med Microbiol 2006; 24: 52-54.

15. Viereck MJ, Chalouhi N, Krieger DI, Judy KD. Cryptococcal ventriculoperitoneal shunt infection. J Clin Neurosci 2014; 21 : 2020-2021.

16. Uçkay I, Pittet D, Vaudaux P, Sax H, Lew D, Waldvogel F. Foreign body infections due to Staphylococcus epidermidis. Ann Med 2009; 41: 109-119.
17. Driscoll JA, Brody SL, Kollef MH. The epidemiology, pathogenesis and treatment of Pseudomonas aeruginosa infections. Drugs 2007; 67: 351-368.

18. Baghdadi J, Hemarajata P, Humphries R, Kelesidis T. First report of ventriculoperitoneal shunt infection due to Cyberlindnera fabianii. Case Rep Infect Dis 2015; 2015: 630816.

19. Rangarajan K, Das CJ, Kumar A, Gupta AK. MRI in central nervous system infections: A simplified patterned approach. World J Radiol 2014; 6: 716-725.

20. Long W, Yuan J, Liu J, Liu J, Wu M, Chen X, et al. Multidrugresistant brain abscess due to Acinetobacter baumannii ventriculitis cleared by intraventricular and intravenous tigecycline therapy: A case report and review of literature. Front Neurol 2018; 9: 518.

21. Bang JH, Cho KT. Rapidly progressive gas-containing lumbar spinal epidural abscess. Korean J Spine 2015; 12: 139-142.

22. Saini AG, Rathore V, Ahuja CK, Chhabra R, Vaidya PC, Singhi P. Multiple brain abscesses due to Enterobacter cloacae in an immune-competent child. J Infect Public Health 2017; 10: 674-677.

23. Iwasaki Y, Inokuchi R, Harada S, Aoki K, Ishii Y, Shinohara K. Bacterial meningitis caused by hypervirulent Klebsiella pneumoniae capsular genotype K54 with development of granuloma-like nodal enhancement in the brain during the subacute phase. Intern Med 2017; 3: 373-376.

24. Majumdar M, Simes DC, Prabha RD. Cerebritis: an unusual complication of Klebsiella pneumoniae. Indian J Crit Care Med 2009; 13: 37-40.

25. Chauhan S, Noor J, Yegneswaran B, Kodali H. Enterobacter meningitis and challenges in treatment. J Clin Diagn Res 2016; 10: OD10-OD11.

26. Noguchi T, Nagao M, Yamamoto M, Matsumura Y, Kitano T, Takaori-Kondo A, et al. Staphylococcus epidermidis meningitis in the absence of a neurosurgical device secondary to catheterrelated bloodstream infection: A case report and review of the literature. J Med Case Rep 2018; 12: 106.

27. Miura FK, Andrade AF, Randi BA, Amato VS, Nicodemo AC. Cerebrospinal fluid shunt infection caused by Corynebacterium sp: case report and review. Brain Inj 2014; 28: 1223-1225.

28. Gutierrez-Murgas Y, Snowden JN. Ventricular shunt infections: Immunopathogenesis and clinical management. $J$ Neuroimmunol 2014; 276: 1-8.

29. Okamura Y, Maruyama K, Fukuda S, Horikawa H, Sasaki N, Noguchi A, et al. Detailed standardized protocol to prevent cerebrospinal fluid shunt infection. J Neurosurg 2019; 1: 1-5. 